\title{
A Novel Protection Algorithm for Medium Voltage Collector Feeder In Wind Farm Based On Quadrature Directional Algorithm With Laboratory Investigation
}

\author{
Abd-ellatif Sayed \\ Dep. of Electrical Power and Machine Engineering \\ Faculty of Engineering, Helwan University \\ Cairo, Egypt \\ elptef2006@yahoo.com
}

\begin{abstract}
A protection scheme for medium voltage wind farm collector feeder has been presented in this paper. The scheme is based on quadrature directional angle. This scheme is convenient for small WTG with capacity less than 1MVA. It uses the voltage signal at the collector bus and the current signals at each WTG zone to calculate the quadrature directional angle of each zone which connected to protected feeder, any fault occurs at any parallel feeder or at power grid is considered as an external fault. A part of Zafarana wind farm which is connected to $220 \mathrm{kV}$ system of the Egyptian network is simulated by using MATLAB package to verify the proposed protection algorithms. The results covered a wide variety of fault scenarios; these results proved the ability of the proposed algorithm to differentiate between in-zone and out-of-zone faults. To verify the effectiveness of the proposed scheme it is tested in the laboratory by using induction machine, synchronous generator and DC Motor to simulate the power system, the current and voltage transformer are used to transfer the current and voltage signals respectively of the power system to the data acquisition system connected to pc by sampling frequency of $10 \mathrm{kHz}$. Lab- View package is used as software. The experimental results indicate that they are compatible with the simulated results and the proposed technique is stable, reliable, and fast during the discrimination between internal and external faults.
\end{abstract}

Keyword-Wind Farm; Collector Feeder; Hardware Laboratory Experiment; Digital Protection Algorithm. 


\section{INTRODUCTION}

Wind generation and wind farms are becoming an important part of the generating capacity of the modern utility grid. Protection of wind turbines, and wind farm collector systems has been the subject of numerous technical papers over recent years, as wind generation grows more prevalent [1]. The protection of wind farm equipment's must be compatible with the protection of the grid [2]. According to the code requirement, wind farms must continue in operation during external fault in the power grid. Wind farm protection requires phase and neutral over current, under and over voltage, over and under frequency and direction of parallel operation [3].

The medium voltage collector feeder in a wind farm is usually protected by conventional protection strategy, which uses a combination of fuses and reclosers [4]. An overview on the methods applied for fault location in medium voltage collector feeder is presented in [5].The commonly methods used depend on travelling wave, impedance based method and the knowledge based methods (genetic algorithm and artificial neural network). Using of directional over current relay for medium cable feeder and the protection of each wind turbine generator connected to the medium voltage cable feeder is done by high rupture current fuse [6-8]. The study of the coordination time between each relay located in the first section of cable feeder is presented in [9], and the relay setting for sectionalizer and recloser is applied in this study, but the relay is not able to discriminate between faults in cable feeder section zones or in wind turbine generator zones.

Determining of the fault location for medium voltage cable feeder based on impedance calculation (distance relay) is presented in [10-11]. The wavelets and discrete wavelet transform in power system network include medium voltage feeder connected with induction generators in wind farm is used to identify the faulted feeder from healthy feeder [12-14]. The polarity of the detail level of residual voltage and detail level of residual current during two cycles are used to discriminate between the internal and external ground faults in the collector feeder [15], but not applicable for line to line and symmetrical three phase short circuit.

The polarity of the impedance of the average value of residual current and voltage with short time window during the fault is also used for collector feeder protection [16].

The effect of lightning on the wind farm which the wind farm collector feeders that transmitted with overhead transmission lines is studied in [17], and the lightening protection is presented in it. The previous protection schemes suffer from some drawback such as inability to discriminate between faults occurring at wind turbine generator (WTG) and faults occurring at the medium voltage cable. It also suffers from inability to isolate faulty cable from WTG side i.e. WTG is feeding the fault

In this paper a protection scheme has been presented based on quadrature directional angle by using the voltage signal of the collector bus and the current signals of each WTG zone to calculate the quadrature directional angle of the protected collector feeder and each WTG zone which are connected to this feeder achieve discrimination between internal faults at collector feeder or at faulted WTG zone and external faults at any parallel feeder or at power grid. The proposed protection algorithms performance is verified by carrying out various fault scenarios using MATLAB package. To verify the effectiveness of the proposed scheme, it is tested in the laboratory. Experimental results show a good agreement with simulation results. 


\section{THE SYSTEM UNDER STUDY}

A part of Zafarana farm connected to Egypt network is used to study the proposed protection algorithm. Zafarana site is divided into 8 zones with different types of wind generators. Each zone is connected to the grid through three or four feeders. The power is transferred to grid through five transformers with rating 125MVA for three winding transformers and 75MVA for two winding transformers as shown in Figure 1 [18-19].

Figure 2 shows the wind farm system model that proposed for the WTGs and cable feeders. The relay uses signals from current transformers located at cable feeder and WTG zones, and voltage transformer at the collection bus.

Figure- 3 shows the complete data collections for the proposed technique for relay feeder- 1 and relay feeder-2.

\section{PROPOSED PROTECTION ALGORITHM}

The directional overcurrent, generally are based on the phase relationship of $\mathrm{V}$ and I. The fault direction is discriminated using quadrature directional angle between measured current and voltage signals from the end of cable feeder at the collection bus and the currents signals measured at each wind turbine generator zone to distinguish between feeder fault (internal faults) and grid fault or faults on any other parallel feeders connected to the collection bus (external fault).

The phase directional relaying quadrature polarizing is used to calculate the directional angle; this means that the polarizing voltage for a fault involving " $\mathrm{A}$ " phase current is voltage $\mathrm{BC}, \mathrm{B}$ phase current is polarized by voltage $C A$, and $C$ phase current is polarized by voltage $A B$ [20]. The term "quadrature" is defined as one quarter or one fourth. In our case the quadrature voltage position on a vector wheel is one fourth of 360 degrees as shown in Figure 4.

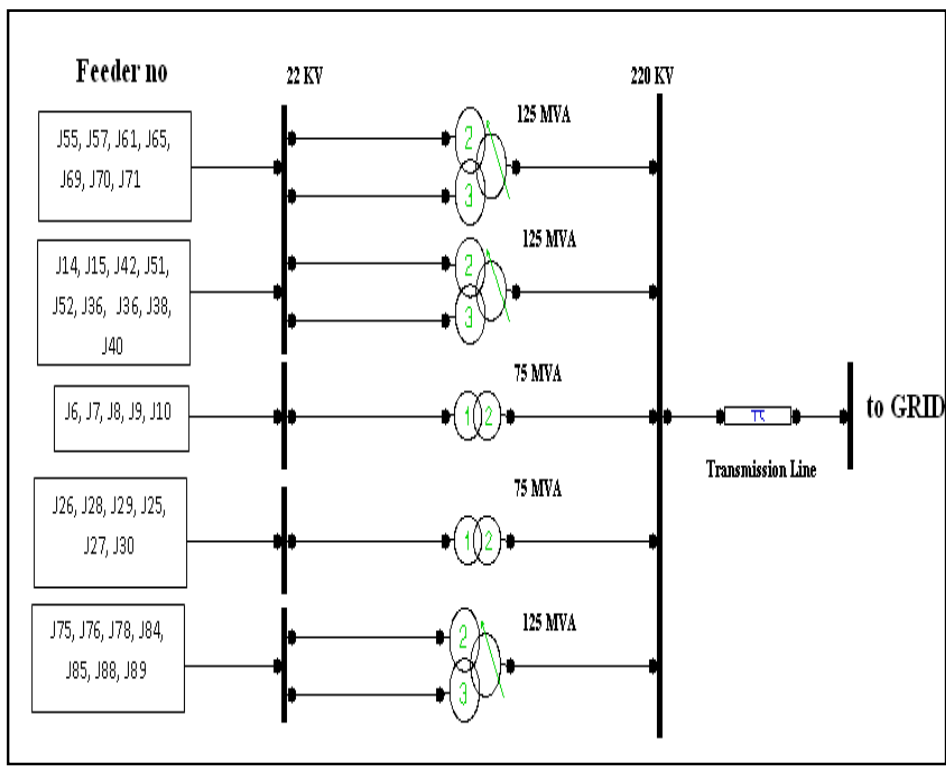

Figurer-1 Zafarana farm model 
A. S. Abd-Elatif / Engineering Research Journal 165(March 2020) E14-E31

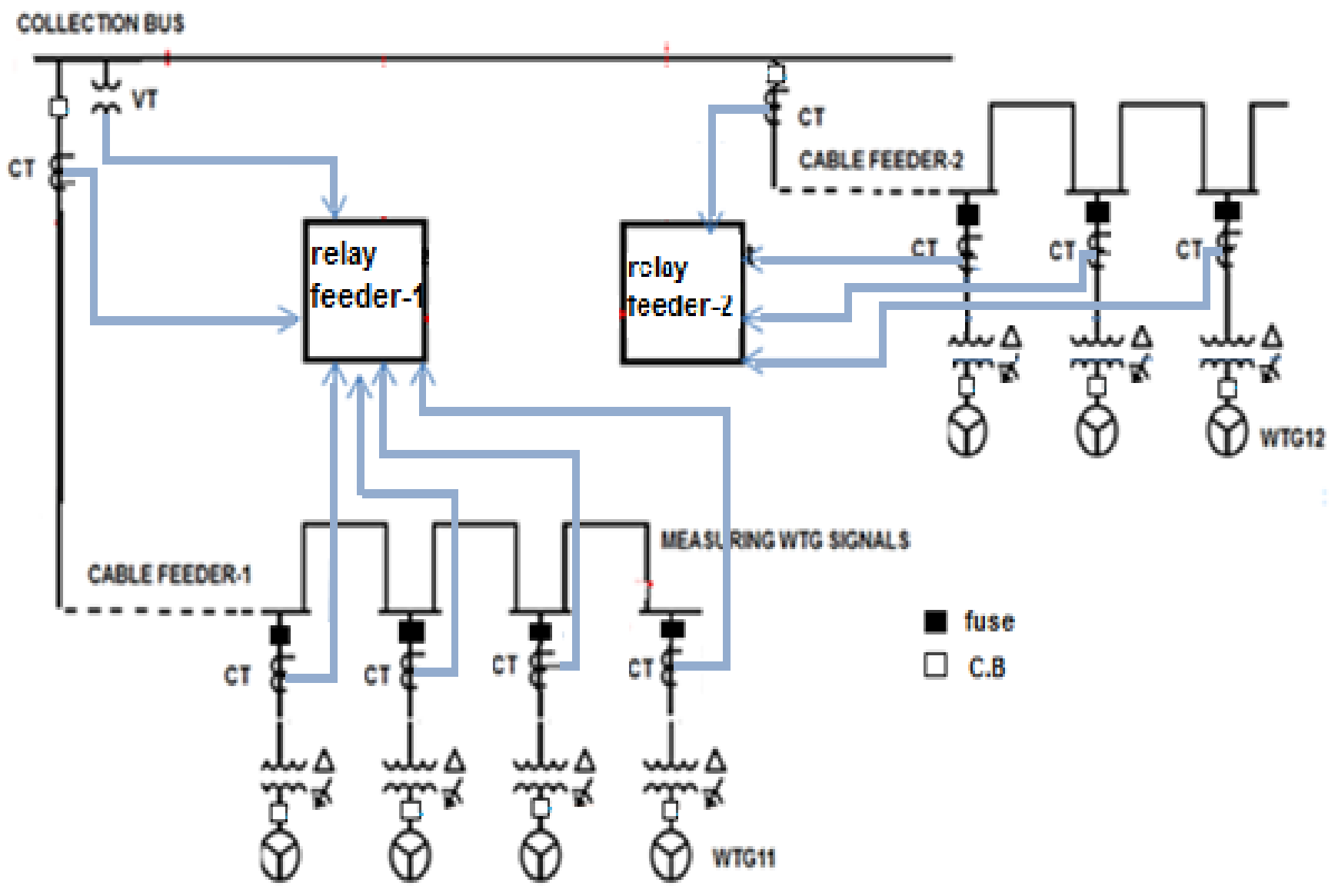

Figure-2 Proposed protection zones
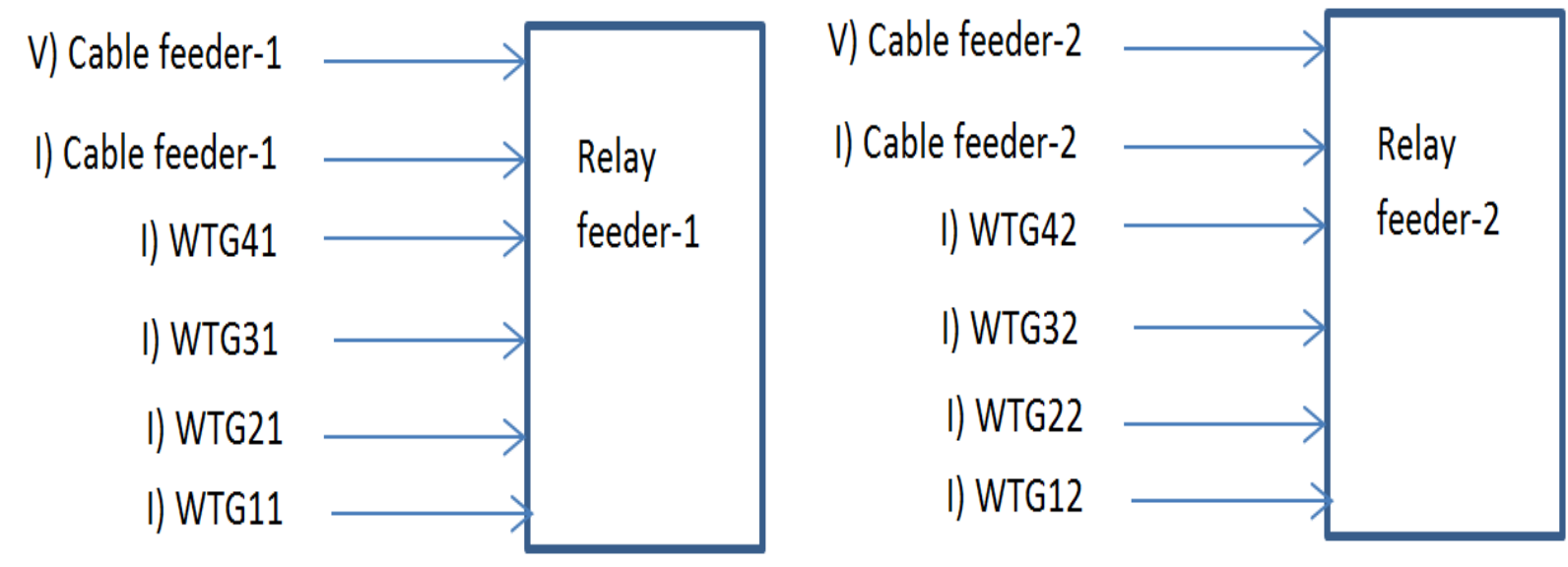

Figure-3 Data collection for the proposed technique 


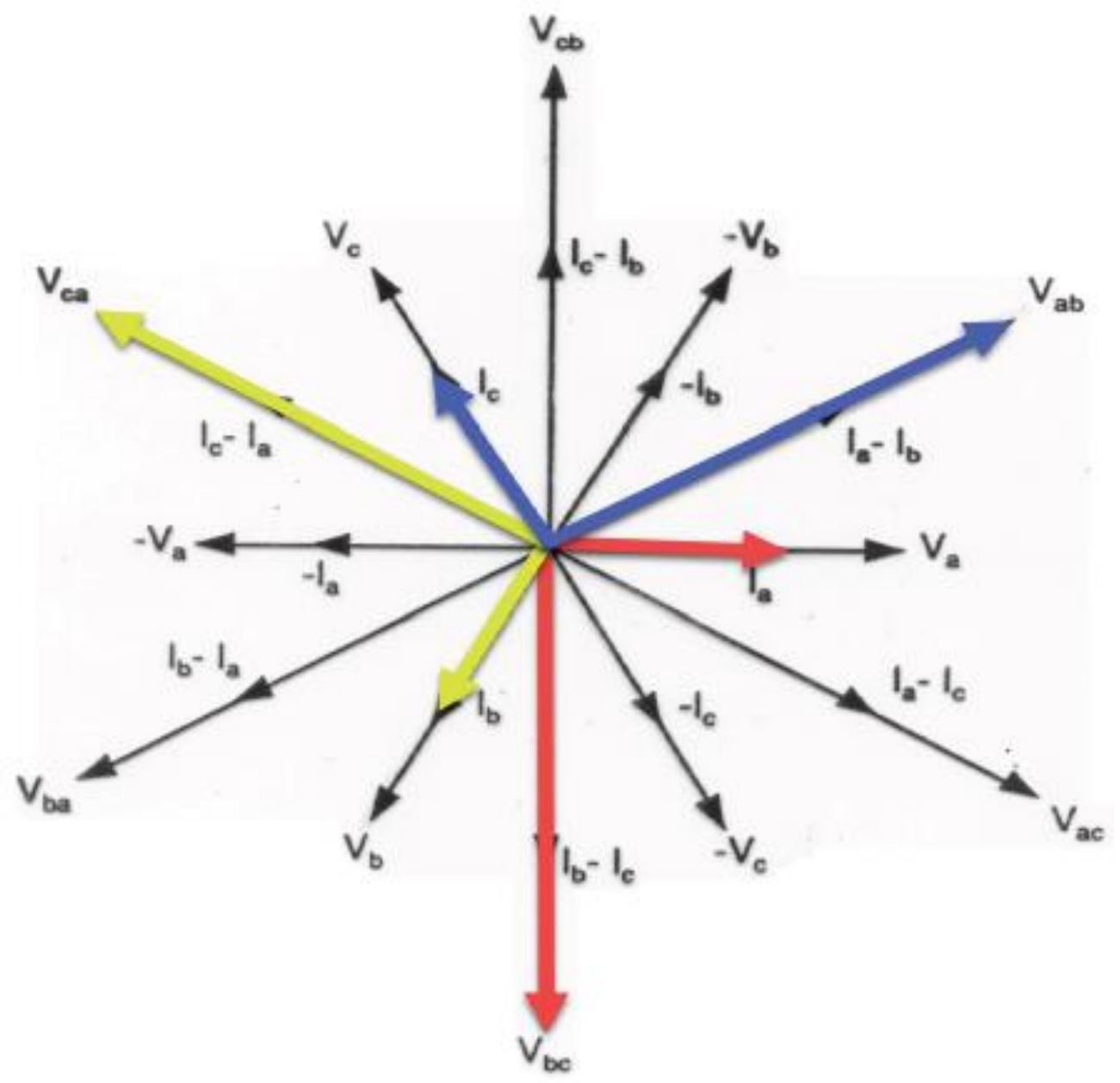

Figure 4 Phases directional relaying quadrature polarizing

The angle setting is commonly referred to as the maximum torque angle ( MTA). For instance, $\mathrm{I}_{\mathrm{a}}$ could be compared to $\mathrm{V}_{\mathrm{ab}}, \mathrm{V}_{\mathrm{ca}}, \mathrm{V}_{\mathrm{bn}}$, or $\mathrm{V}_{\mathrm{cn}}$, and the detection algorithm would work, though the quadrature voltage $\mathrm{V}_{\mathrm{bc}}$ gives the most independence of the voltage signal from the effects of an $\mathrm{AG}, \mathrm{AB}$, or $\mathrm{AC}$ fault as shown in Figure 5.
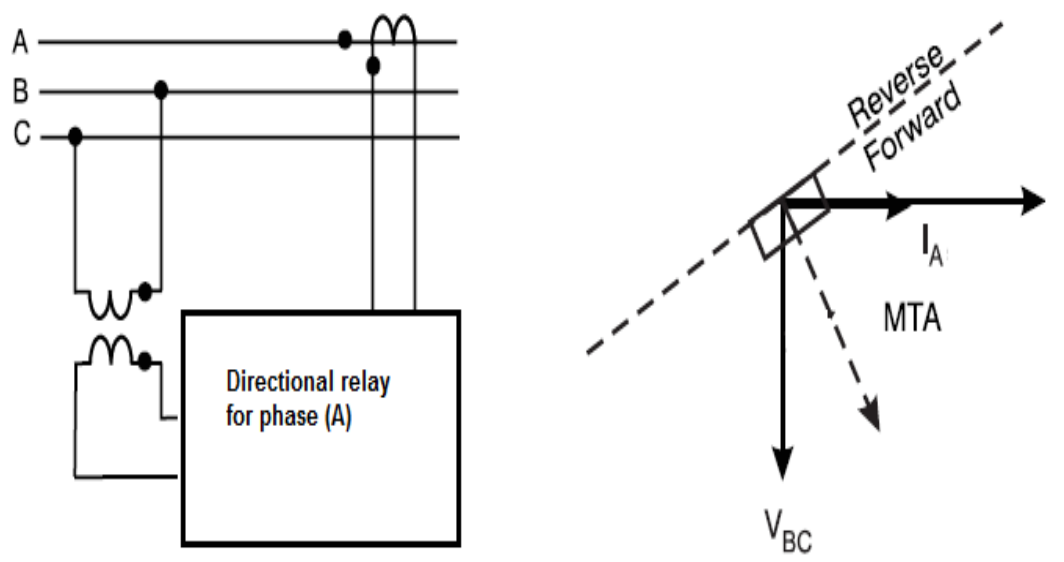

Figure 5 Directional relay signals for phase (a) and its maximum torque angle. 
During faults the current signals have harmonic contents, these harmonic contents effect on the calculation of the angle, and the relay may maloperation, so these harmonic contents must be taken into consideration for directional angle calculation,

To eliminate these harmonic contents, Discrete Fourier Transform (DFT) tool is used for the direction identification.

The DFT for function $\mathrm{X}(\mathrm{f})$ is in the form.

$$
X\left(f_{n}\right)=\frac{2}{N} \sum_{n=0}^{N-1} A \cos \left(2 \pi \frac{n}{N}+\theta\right) e^{-j 2 \pi \frac{n}{N}}
$$

Where $\mathrm{N}$ is the number of samples per the power cycle, $\mathrm{n}=0, \ldots \ldots, \mathrm{N}-1$.

A and $\theta$ are the magnitude and the phase of the signal and $w$ is angular frequency

The equation (1) is derived into real and imaginary terms as in equation (2)

$\mathrm{X}\left(\mathrm{f}_{\mathrm{n}}\right)=\frac{2}{\mathrm{~N}} \sum_{\mathrm{n}=0}^{\mathrm{N}-1} \mathrm{x}[\mathrm{n}] \mathrm{e}^{-\mathrm{j} 2 \pi \frac{\mathrm{n}}{\mathrm{N}}}=$

$\frac{2}{N} \sum_{n=0}^{N-1} x[n] \cos 2 \pi \frac{n}{N}+j \frac{2}{N} \sum_{n=0}^{N-1}-x[n] \sin 2 \pi \frac{n}{N}$

Where:

$\mathrm{x}_{\mathrm{r}}$ is the real filter $=\frac{2}{\mathrm{~N}} \cos \left(2 \pi \frac{\mathrm{n}}{\mathrm{N}}\right)$

$\mathrm{x}_{\mathrm{i}}$ is the imaginary filter $=-\frac{2}{\mathrm{~N}} \sin \left(2 \pi \frac{\mathrm{n}}{\mathrm{N}}\right)$

Phasor's amplitude and angle can be computed by (3) and (4), respectively.

$$
\begin{aligned}
& \mathrm{A}=\sqrt{\mathrm{x}_{\mathrm{r}}{ }^{2}+\mathrm{x}_{\mathrm{i}}{ }^{2}} \\
& \angle \theta=\arg \left(\mathrm{x}_{\mathrm{r}}+\mathrm{jx}_{\mathrm{i}}\right)
\end{aligned}
$$

So the phasor's angle of voltage and current signals can be computed by (5) and (6), respectively.

$$
\begin{aligned}
& \angle \theta_{V_{-} b c}=\arg \left(\mathrm{xv}_{\mathrm{r}}+\mathrm{jxv}_{\mathrm{i}}\right) \\
& \angle \theta_{I_{-} a}=\arg \left(\mathrm{xi}_{\mathrm{r}}+\mathrm{jxi}_{\mathrm{i}}\right)
\end{aligned}
$$

Where

$\mathrm{xv}_{\mathrm{r}}$ : real filter of voltage $\left(V_{b c}\right)$

$\mathrm{xv}_{\mathrm{i}}$ : imaginary filter of voltage $\left(V_{b c}\right)$

$\mathrm{xi}_{\mathrm{r}}$ : real filter of current $\left(I_{a}\right)$ 
$\mathrm{xi}_{\mathrm{i}}$ : imaginary filter of current $\left(I_{a}\right)$

So the quadrature angle $\angle \theta_{x}$ can be computed by equations (7:9) for each phase.

$$
\begin{aligned}
& \angle \theta_{a}=\angle \theta_{V_{-} b c}-\angle \theta_{I_{-} a} \\
& \angle \theta_{b}=\angle \theta_{V_{-} c a}-\angle \theta_{I_{-} b} \\
& \angle \theta_{c}=\angle \theta_{V_{-} a b}-\angle \theta_{I_{-} c}
\end{aligned}
$$

The directional relay measures the changing in the quadrature angle $\angle \theta_{x}$, during normal operation the angle not change, while the changing of it in case of external faults is less than $90^{\circ}$, but in case of internal faults the angle will be changed by more than $90^{\circ}$, where the direction of power is reversed with respect to the protection relay as shown in Fig. 6.

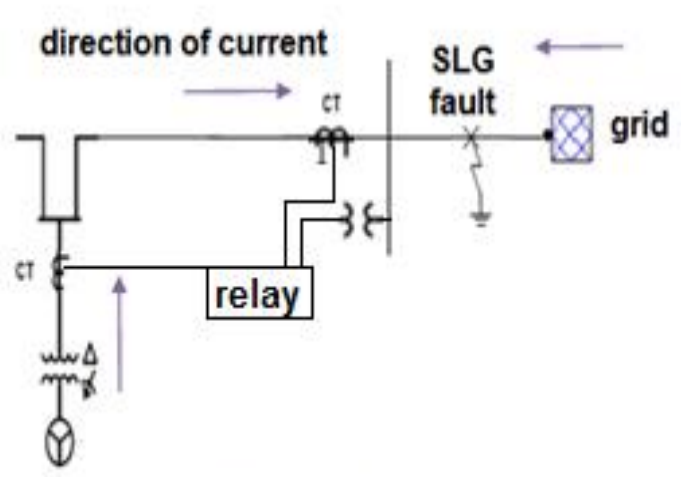

external fault

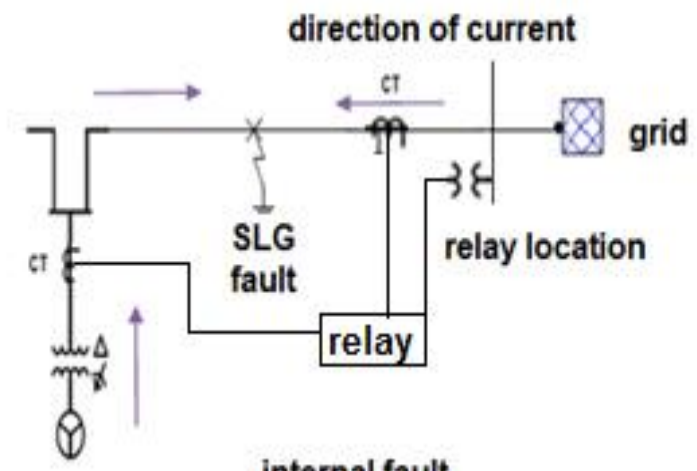

internal fault

Figure 6 Current directions during external and internal fault

Figure 7 shows the relay processing angle and the relay logic gate used to determine the fault cases as shown in table 1 .

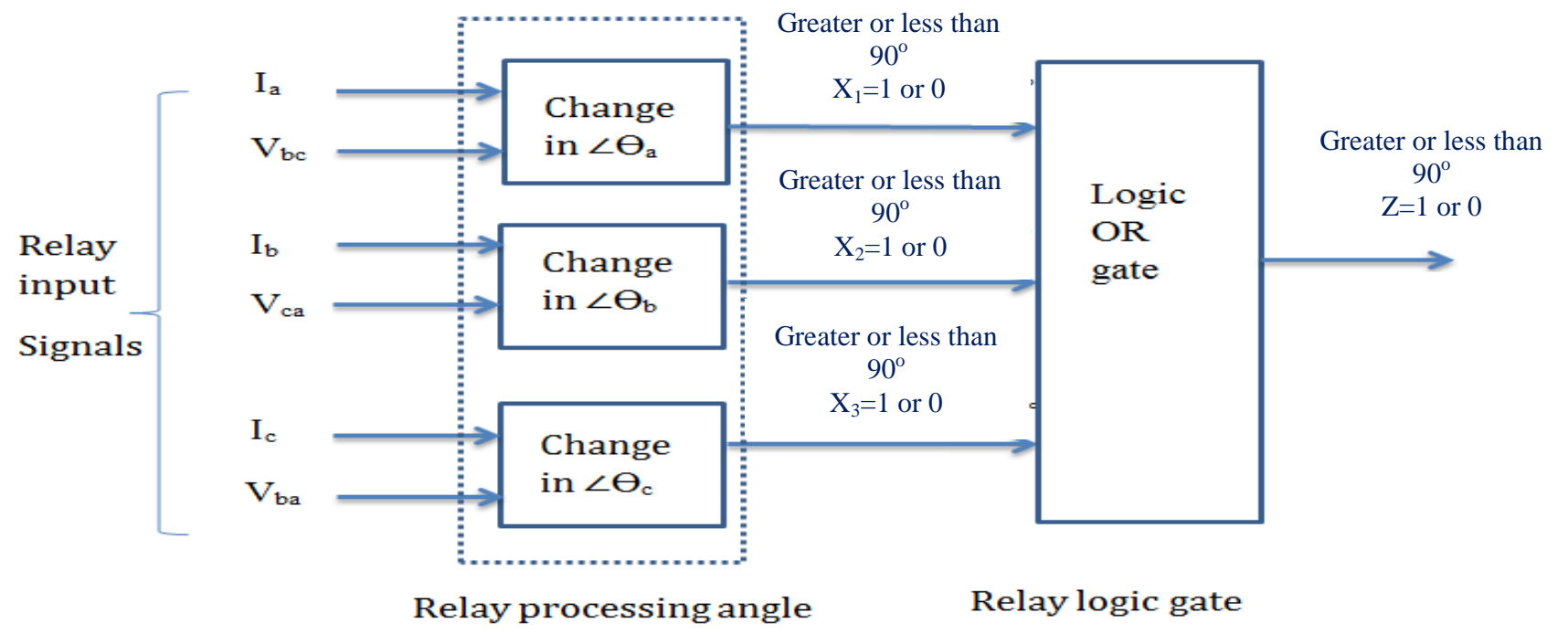

Figure-7 relay processing angle and relay logic gate 
Table-1 the relay logic output

\begin{tabular}{|c|c|c|c|c|}
\hline Operation mode & $\mathbf{X}_{\mathbf{1}}$ & $\mathbf{X}_{\mathbf{2}}$ & $\mathbf{X}_{\mathbf{3}}$ & $\mathbf{Z}$ \\
\hline Normal or External faults & $\mathbf{0}$ & $\mathbf{0}$ & $\mathbf{0}$ & $\mathbf{0}$ \\
\hline A-G & $\mathbf{1}$ & $\mathbf{0}$ & $\mathbf{0}$ & $\mathbf{1}$ \\
\hline B-G & $\mathbf{0}$ & $\mathbf{1}$ & $\mathbf{0}$ & $\mathbf{1}$ \\
\hline C-G & $\mathbf{0}$ & $\mathbf{0}$ & $\mathbf{1}$ & $\mathbf{1}$ \\
\hline A-B/G & $\mathbf{1}$ & $\mathbf{1}$ & $\mathbf{0}$ & $\mathbf{1}$ \\
\hline A-C/G & $\mathbf{1}$ & $\mathbf{0}$ & $\mathbf{1}$ & $\mathbf{1}$ \\
\hline B-C/G & $\mathbf{0}$ & $\mathbf{1}$ & $\mathbf{1}$ & $\mathbf{1}$ \\
\hline A-B-C & $\mathbf{1}$ & $\mathbf{1}$ & $\mathbf{1}$ & $\mathbf{1}$ \\
\hline
\end{tabular}

The proposed algorithm is composed of the two stages as shown in the flowchart in figure 8 .

- Stage1, the measured currents and voltages from the end of cable feeder at the collection bus are used to determine the fault direction. The fault direction is discriminated using the directional angle, to distinguish between feeder faults (internal faults), grid and another parallel feeder faults (external fault).

- Stage 2, the measured data currents at each wind turbine generator and voltage at the collection bus is used also to calculate the fault direction to distinguish cable feeder faults from wind turbine generator fault.

Figure -9 shows the schematic diagram of the studied system with relaying signal and fault location. Each feeder composed multi WTG, but the measurement is taken for only 4 wind turbines that have same operation condition.

Table- 2 shows the functions that the feeder protection relay should have action for all faults (F1F10) in Figure-10.

Table-2 the relay actions for all fault cases

\begin{tabular}{|c|c|}
\hline $\begin{array}{c}\text { Fault } \\
\text { location }\end{array}$ & Relay action \\
\hline F1-F3 & WTG zones, isolate faulted WTG zone \\
\hline F4-F6 & $\begin{array}{c}\text { Trip action of cable feeder and isolating all } \\
\text { WTGS connected to this feeder }\end{array}$ \\
\hline F7-F10 & No action (external fault) \\
\hline
\end{tabular}

The proposed algorithm with the proposed configuration of the voltage and current transformers locations are suggested to overcome the main drawbacks of the previous works proposed to collector feeder protection that is inability to discriminate between faults occurring at wind turbine generator (WTG) and faults occurring at the medium voltage cable. It also suffers from inability to isolate faulty cable from WTG side i.e. WTG is feeding the fault 
A. S. Abd-Elatif / Engineering Research Journal 165(March 2020) E14-E31

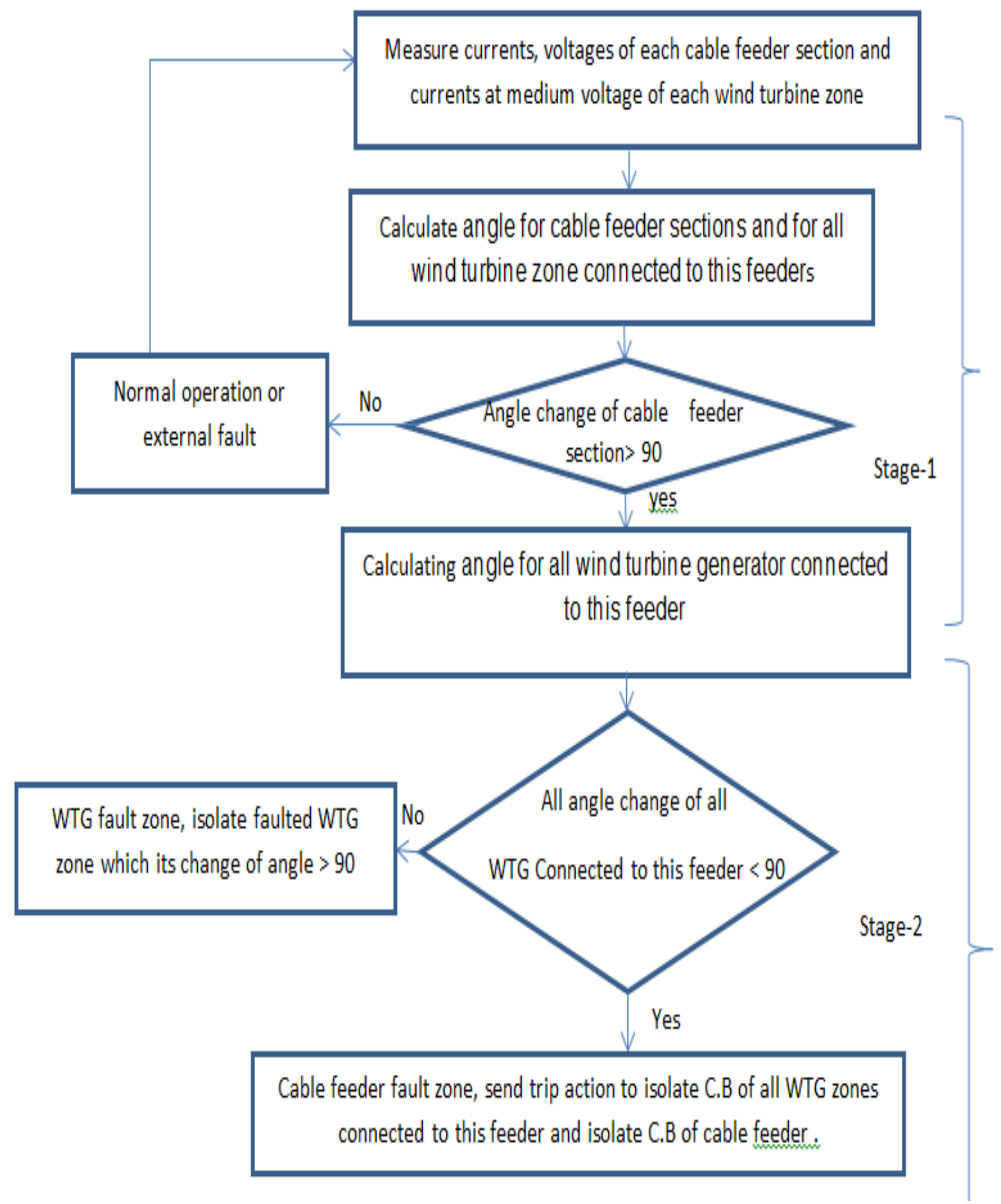

Figure 8 Flow chart for the proposed algorithm 


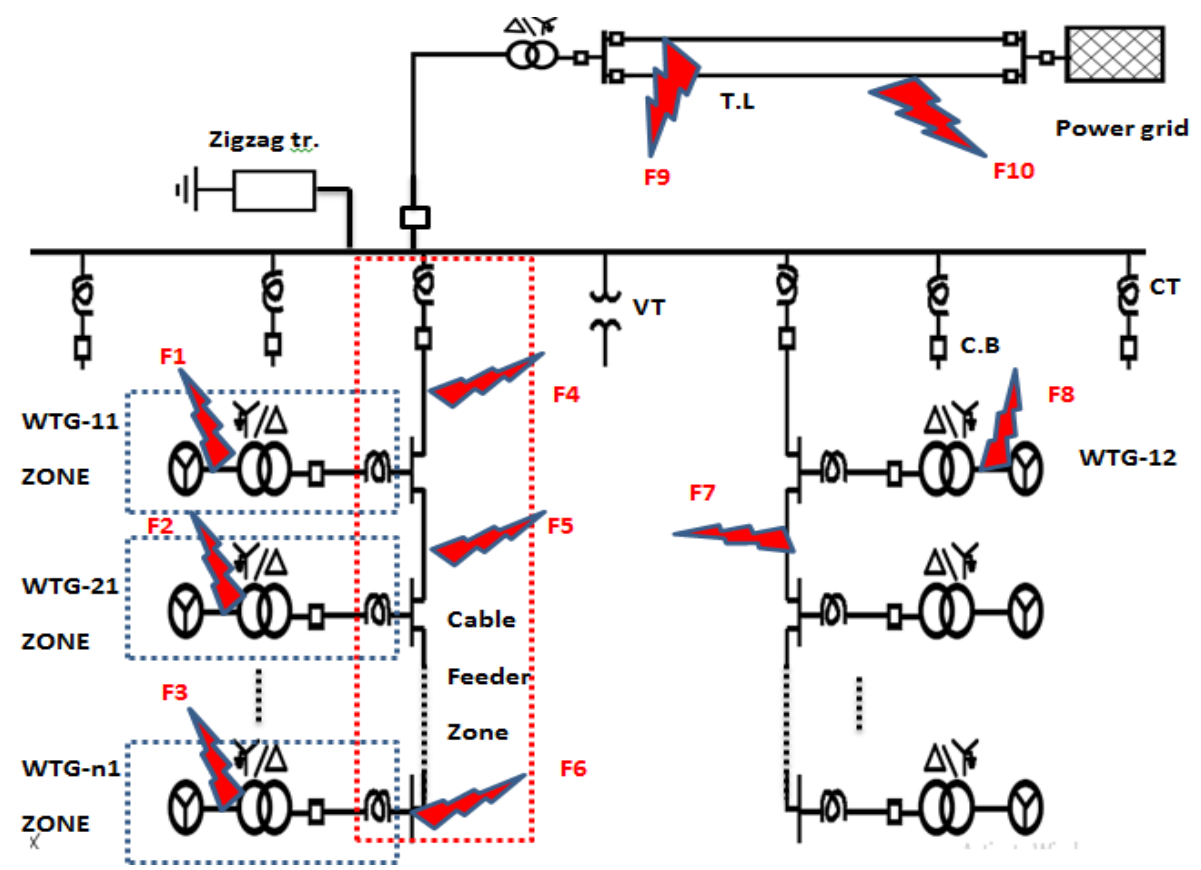

Figure -9 Schematic diagrams for feeder relay

\section{RESULTS AND DISCUSSION}

Figure 10 shows the result of measured angles for collector cable feeder and all WTG connected to this feeder, in case of normal operation the polarized angles between $I_{a}$ with respect to $V_{b c}$ for phase (a), polarized angles between $\mathrm{I}_{\mathrm{b}}$ with respect to $\mathrm{V}_{\mathrm{ca}}$ for phase (b) and polarized angles between $I_{c}$ with respect to $V_{a b}$ for phase (c) as described previously, the results show that the measured angles are constant for all phases for all zones. There are no changes in the calculated angles. The logic relay gate output for polarized directional angles is shown also. The relay logic gate output is equal zero for all feeder zones, so that the relay will not operate.
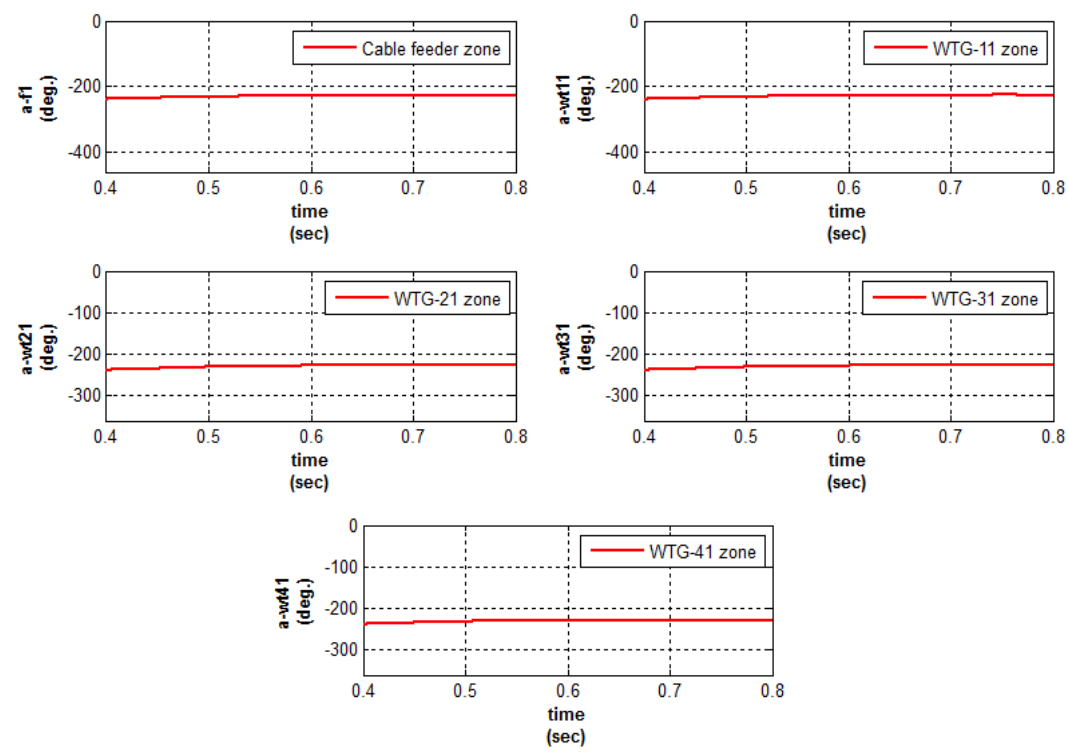

Relay polarized directional angle for phase-a. 
A. S. Abd-Elatif / Engineering Research Journal 165(March 2020) E14-E31
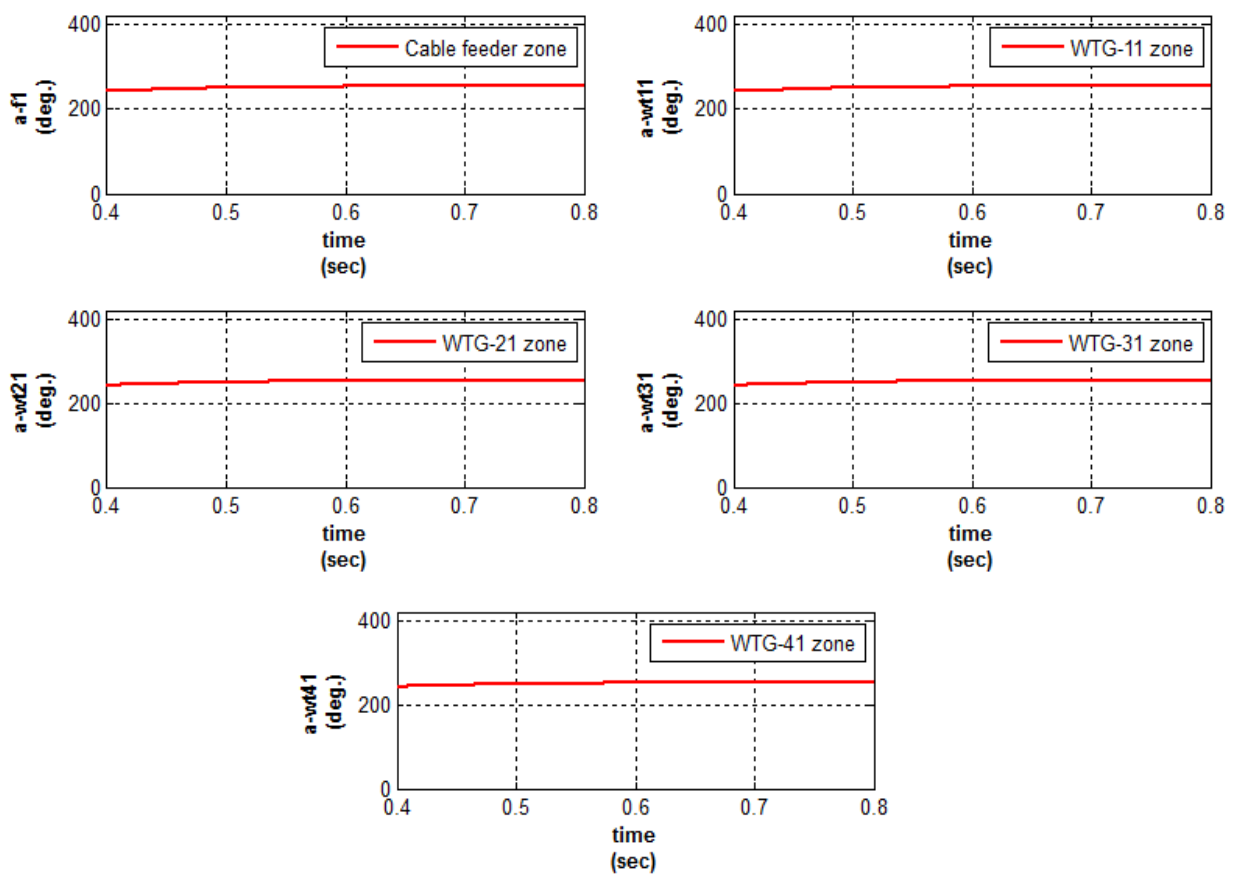

b- Relay polarized directional angle for phase-b.
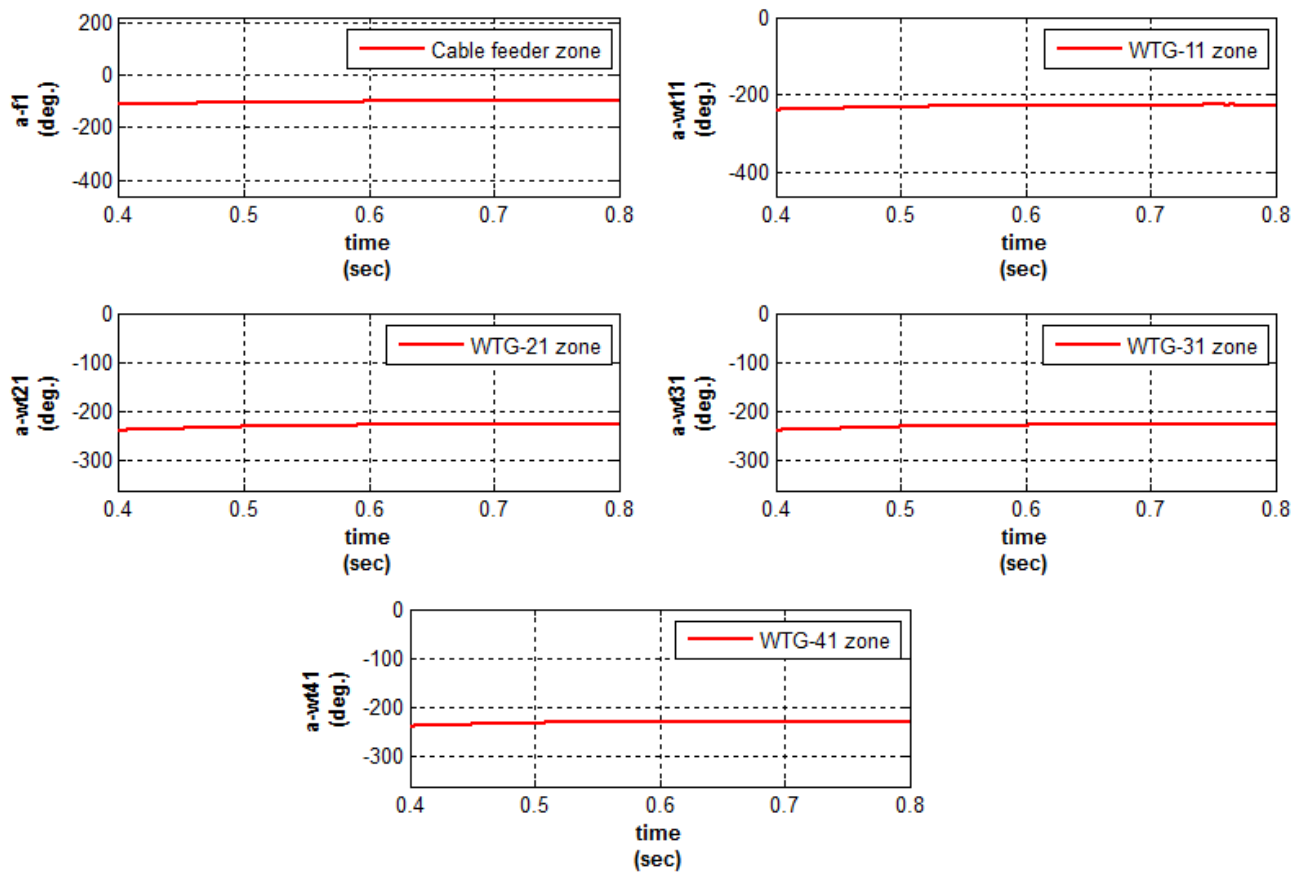

c- Relay polarized directional angle for phase-c. 

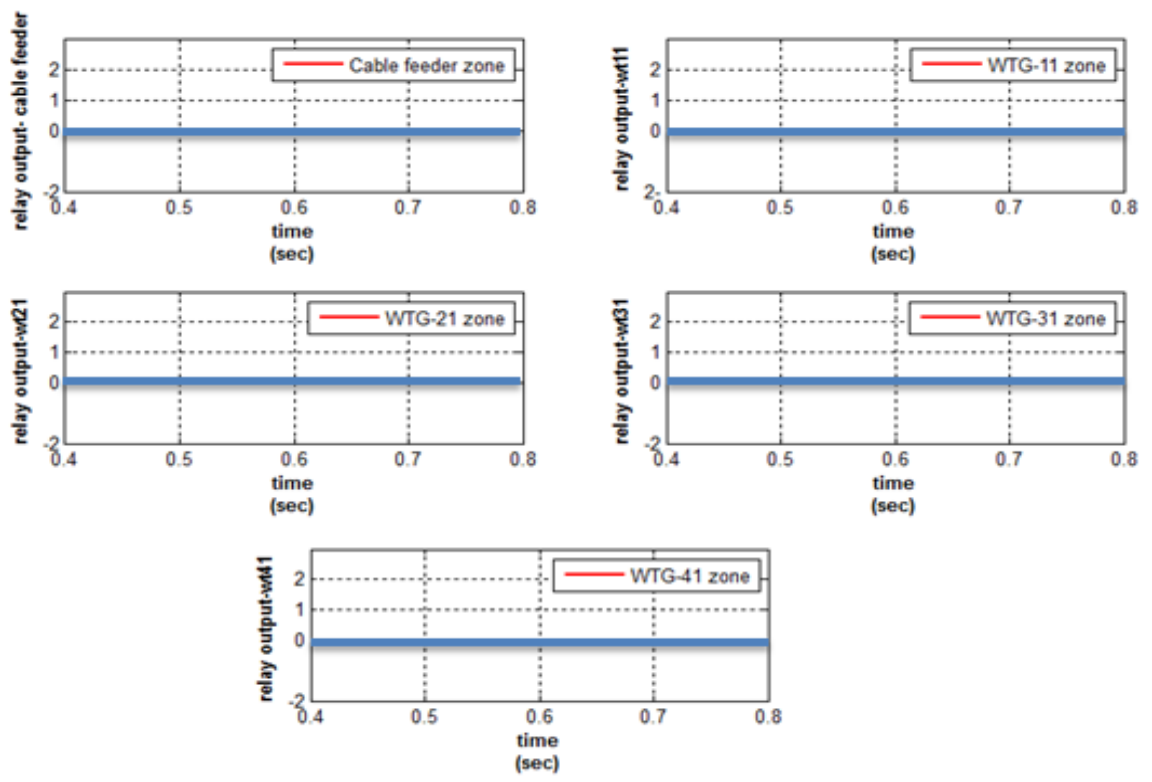

d- Relay logic OR gate output.

Figure 10 Feeder relay response during normal operation

\section{a- Relay response during External Three Phase Fault at WTG-12 ( $\mathbf{F}_{8 \_}$ABCG)}

Figure 11 shows the logic relay gate output for polarized directional angles in case of three phases to ground external fault $\left(\mathrm{F}_{8}\right)$ at WTG-12 at $0.5225 \mathrm{~s}$, the results are calculated for 400 samples per cycle. The logic OR gate output for polarized directional angles. The relay logic gate output is equal zero for all feeder zones, so the relay is not operating for this external fault.
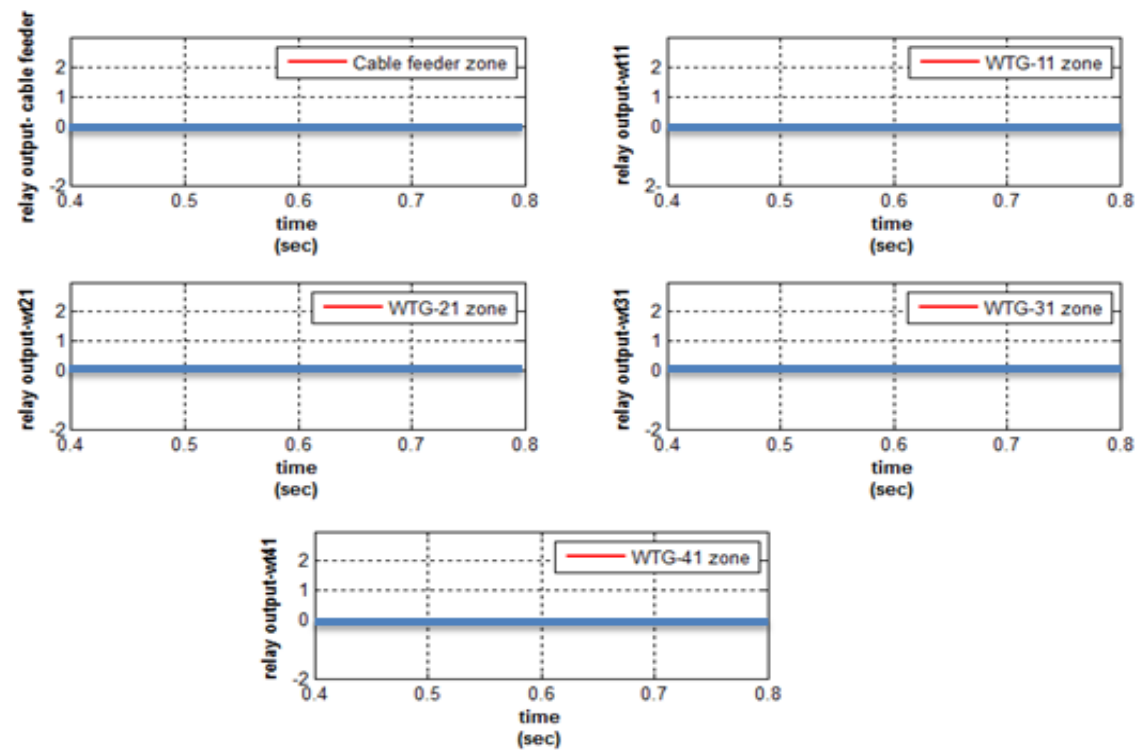

Figure 11 Relay logic OR gate output for external fault 


\section{b- Relay response during Internal Single Line to Ground Fault at WTG11 (F_AG)}

Figure 12 shows the logic relay gate output for polarized directional angles in case of single line to ground internal fault $\left(\mathrm{F}_{1}\right)$ at WTG-11 at $0.5 \mathrm{~s}$, the results are calculated for 400 samples per cycle. The logic OR gate output for polarized directional angles. The relay logic gate output is equal one for cable feeder-1 and WTG11 zones, so the relay isolates WTG11 connected to this feeder, this indicate that the fault at WTG11.
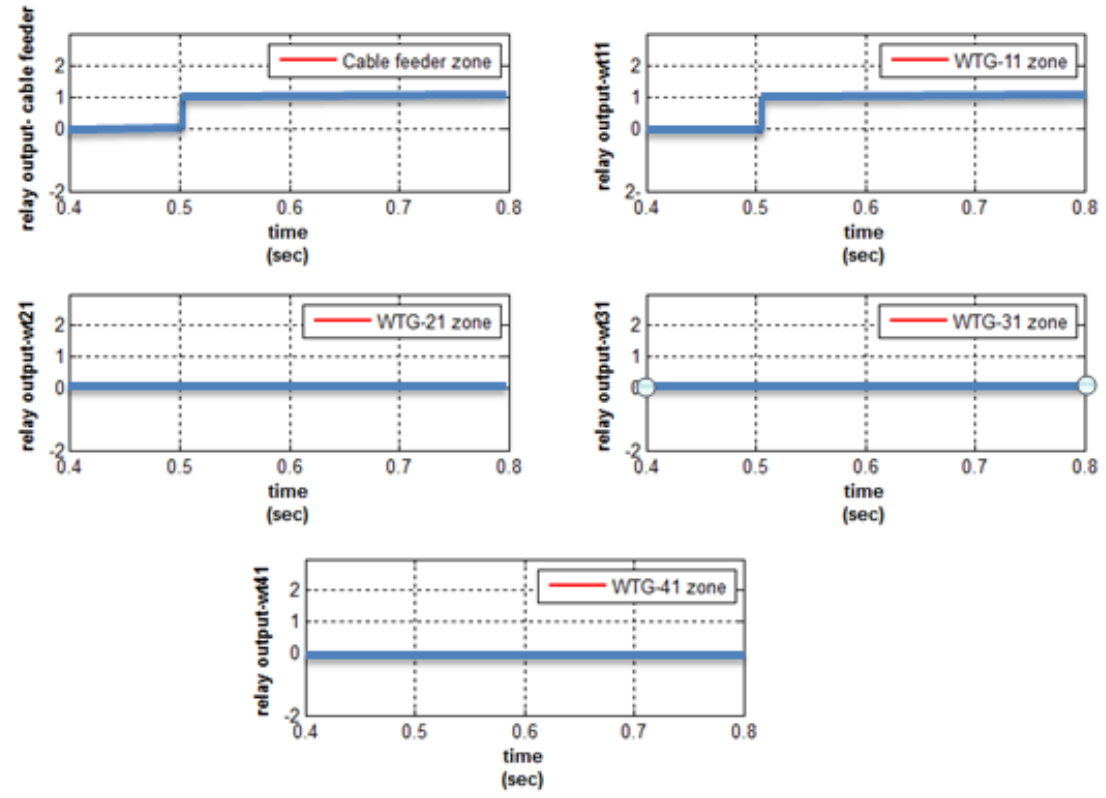

Figure 12 Feeder relay response during SLG fault occurs at WTG11 (F1_AG)

\section{c- Relay response during Internal Line to Line Fault at Cable Feeder-1 ( $\left.F_{6} \_A B\right)$}

Figure 13 shows the logic OR gate output for polarized directional angles for collector cable feeder and all WTG connected to this feeder, where L-L fault $\left(\mathrm{F}_{6}\right)$ occurs at cable feeder- 1 at $0.5 \mathrm{~s}$, the results are calculated for 400 samples per cycle. The changing of directional angles of all WTGs zones are smaller than 90 degree, but the change of it for cable feeder-1 is greater than 90 degree, so the relay logic gate output is equal one for cable feeder- 1 zone; this means the fault at cable feeder- 1 .

\section{HARDWARE IMPLEMENTATION}

Figure (14) shows the wiring diagram of the laboratory experimental setup, the components of the system as follow,

- Three phase induction motor and three phase synchronous generator.

- 2 DC motor.

- Three current transformers and three voltage transformers.

- Data Acquisition Card with sample rate $10 \mathrm{~K} \mathrm{~Hz}$.

The interface card receives three current signals and three voltage signals from power circuit through current and voltage transformers respectively, the Data Acquisition Card interfaced with pc core i7 processor based Lab-View. 

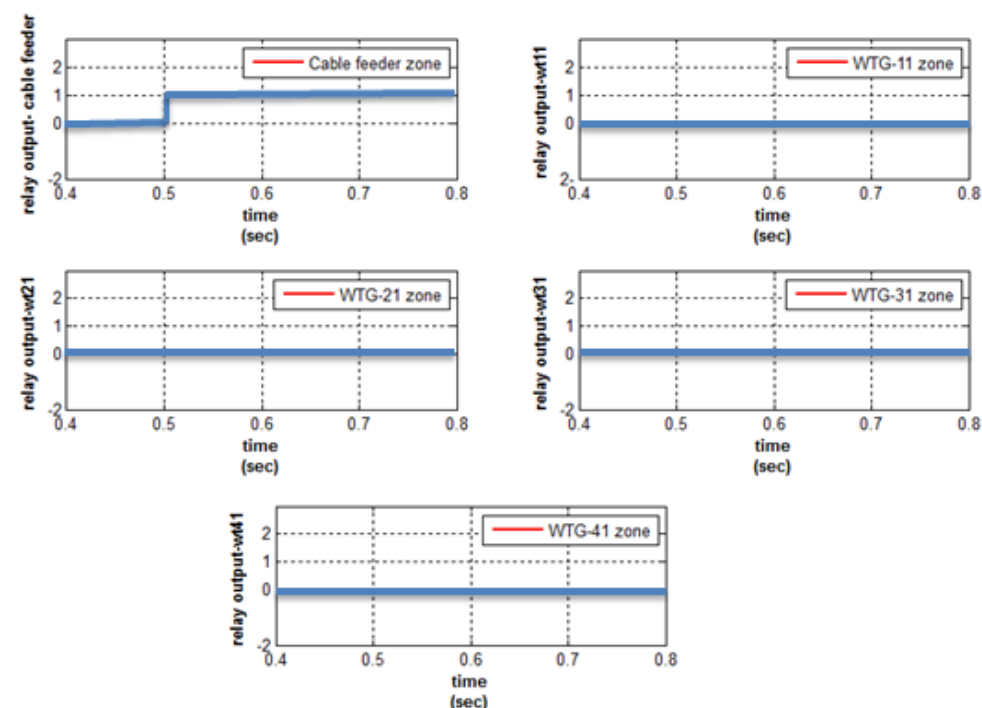

Figure 13 Feeder relay response during Line to Line (L-L) Fault at Cable Feeder-1 (F6_AB)

\section{A. algorithm Implementation}

To implement the proposed technique Lab-View package is used while the used simulation signals in the previous part is instead by real measurement by using current and voltage transformers, the Lab-View is a powerful and flexible instrumentation it used to take measurements, control processes, and analyzing the stored data. Lab View uses a graphical programming language as the $\mathrm{G}$ graphical programming [21].

\section{B. External Single Line to Ground}

Figure-15 shows the phase voltages signals $\left(\mathrm{v}_{\mathrm{a}}, \mathrm{v}_{\mathrm{b}}\right.$ and $\left.\mathrm{v}_{\mathrm{c}}\right)$ and current signals $\left(\mathrm{i}_{\mathrm{a}}, \mathrm{i}_{\mathrm{b}}\right.$ and $\left.\mathrm{i}_{\mathrm{c}}\right)$ during external single line to ground fault at phase (a). The calculated quadrature angle for phase $\mathrm{A}$ is shown also in this figure. The change in the angle is smaller than 90 degree, so the relay is deactivated for this external fault.

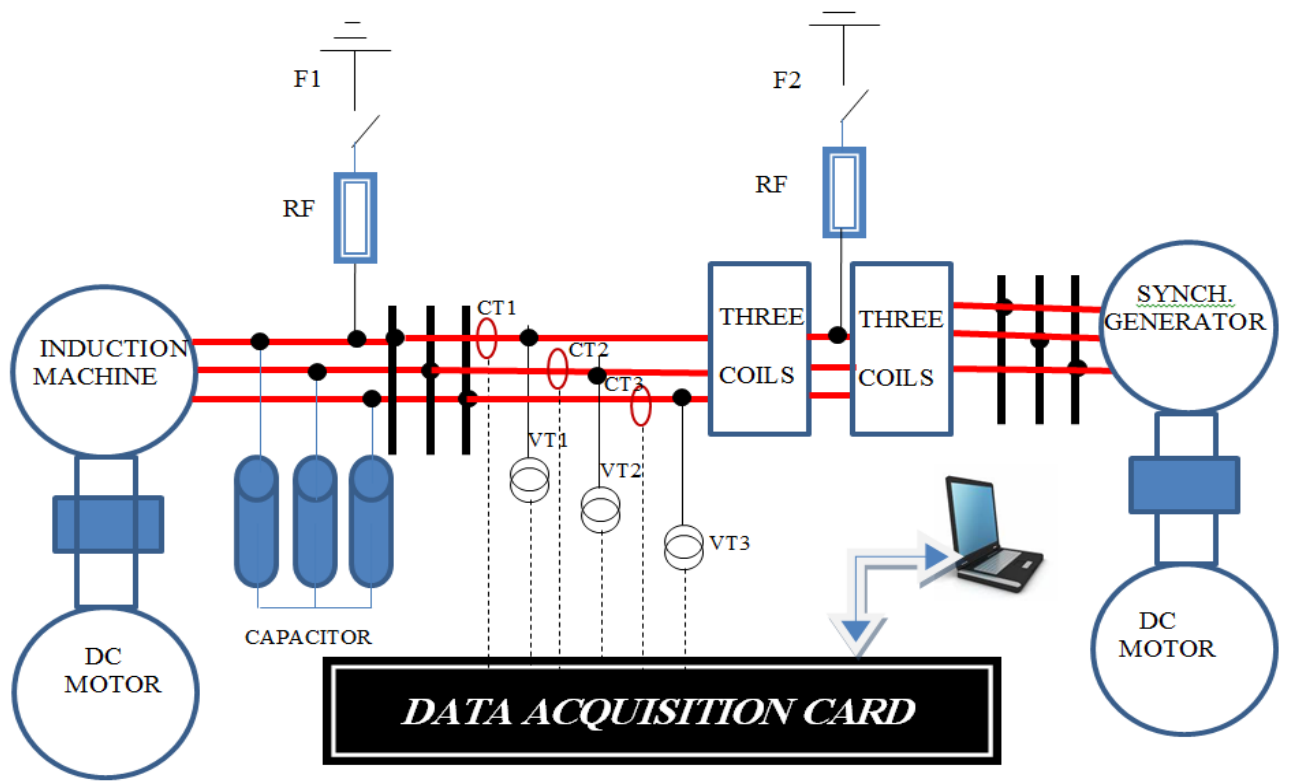

Figure-14 Circuit diagram of the hardware implementation setup. 


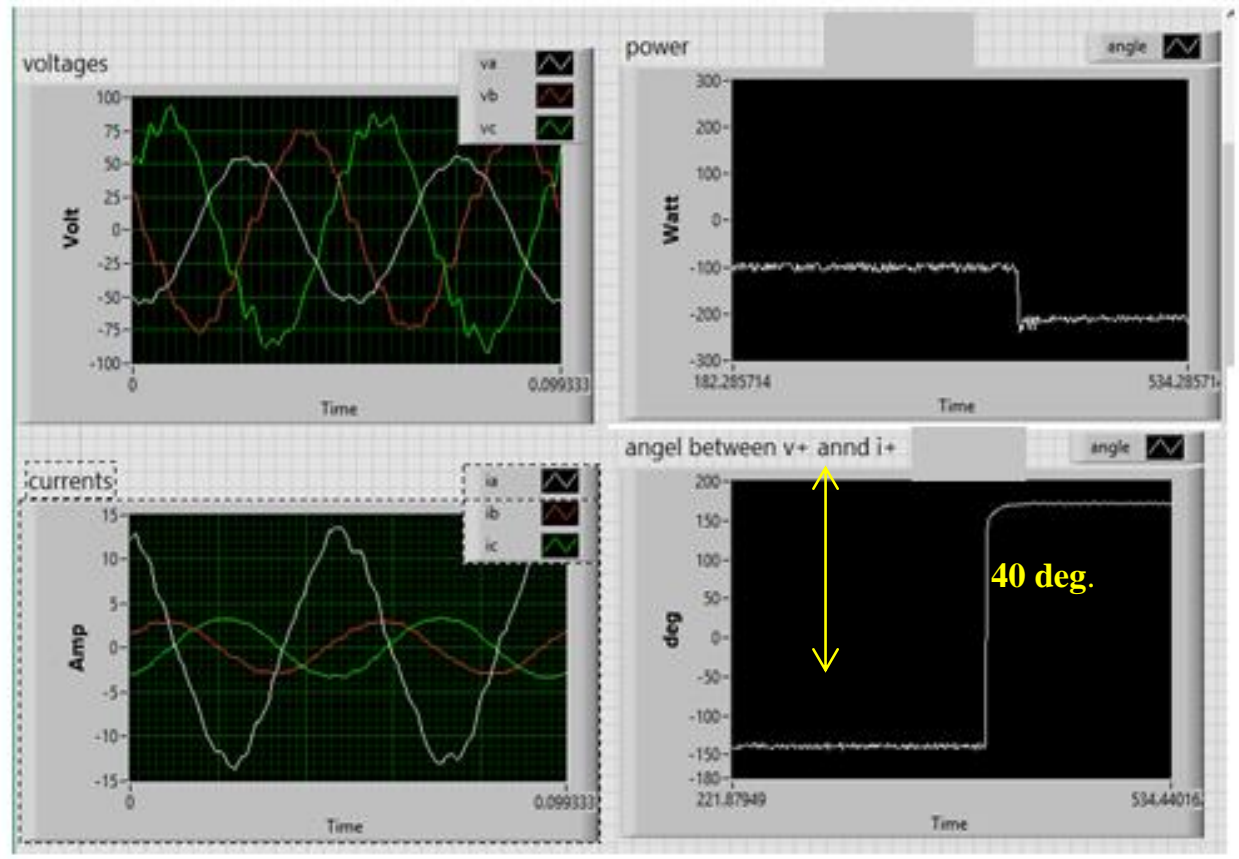

Figure-15 Laboratory result at external single line to ground at phase (a).

\section{Internal Single Line to Ground}

In case of single line to ground internal fault at phase A voltage and current signals are shown in figure 16. The change in the quadrature angle in this case is increased than 90 degree. So the relay will operate to isolate this faulted zone.
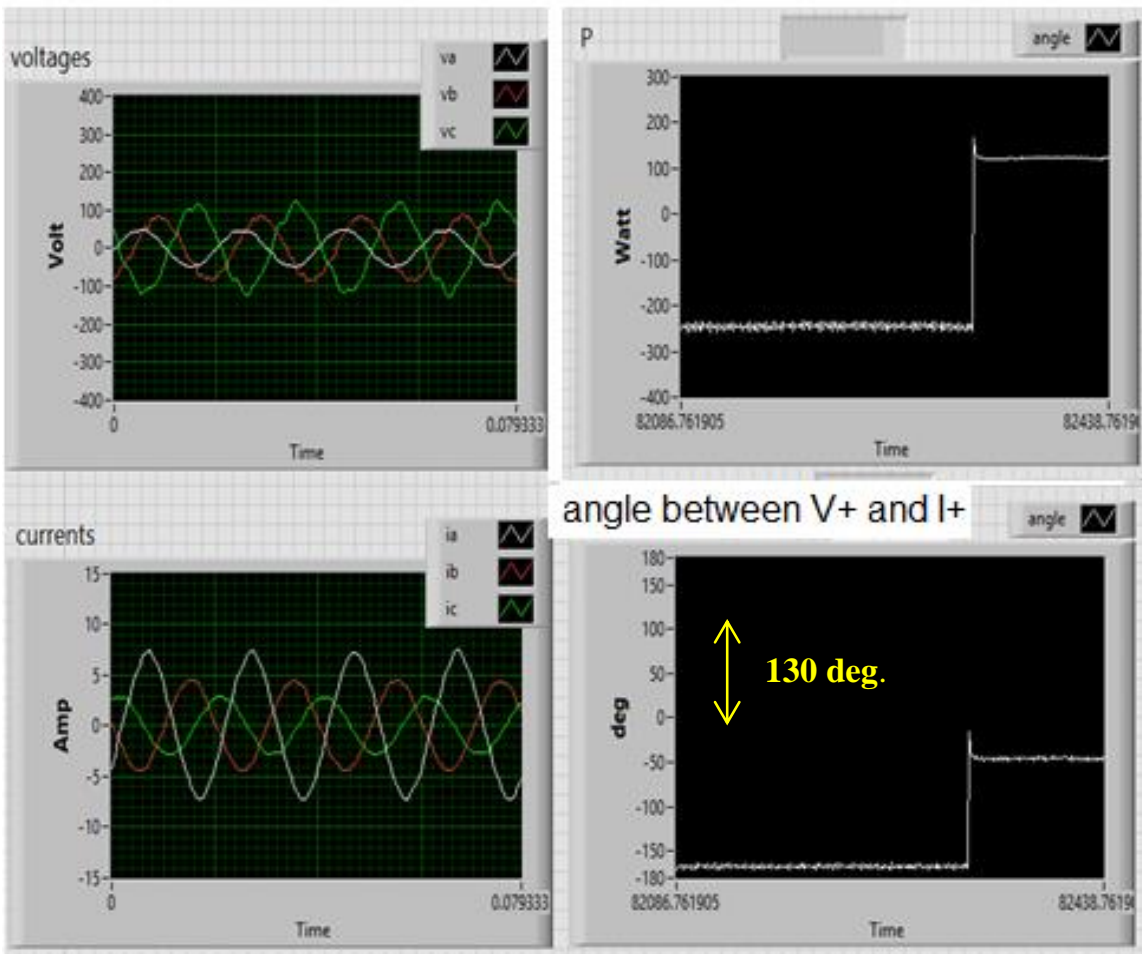

Figure-16 Internal single line to ground fault (A-G) 


\section{Internal Line to Line Fault}

As described in the previous case for single line to ground internal fault the change of quadrature angle in this case is increased than 90 degree. So the relay will operate to isolate this faulted zone, the relay in this case of line to line fault at phases A and B the change of quadrature angle is increased than 90 degree as shown in figure 17. So the relay will operate.

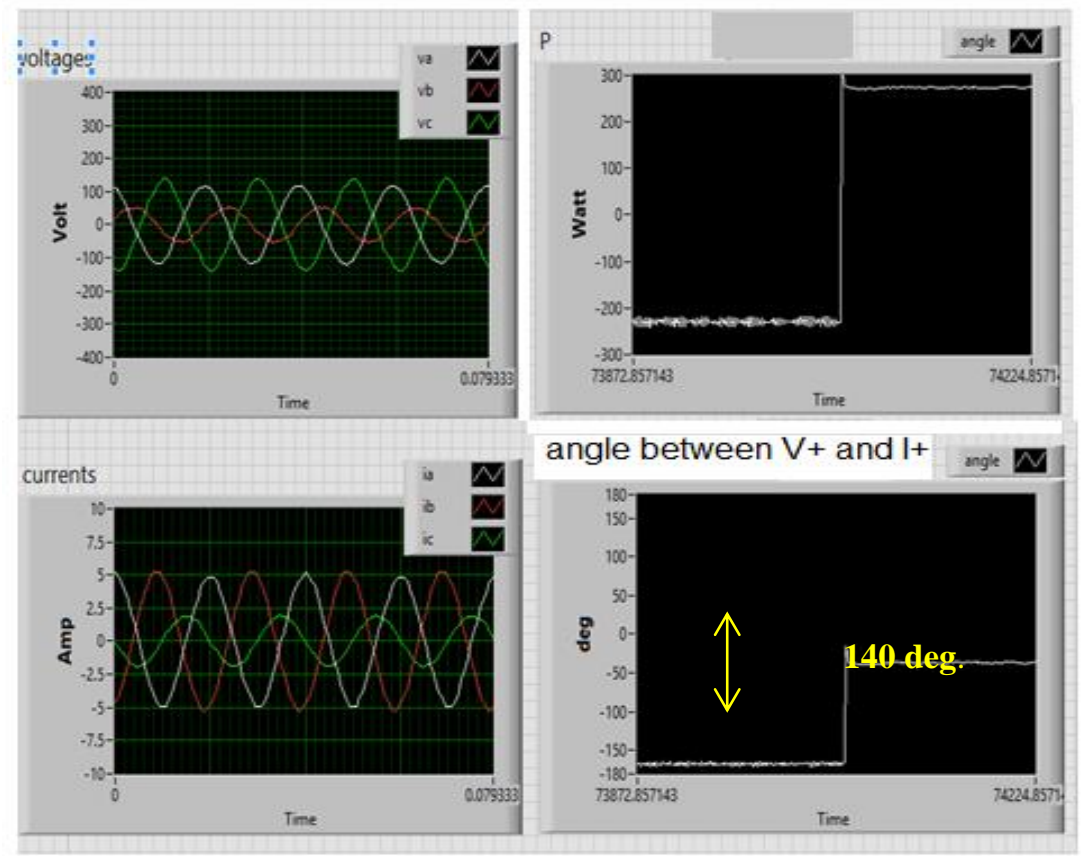

\section{CONCLUSIONS}

Figure-17 Internal line to line fault (A-B)

In this paper a part of Zafarana wind farm is a modeled using MATLAB program. The performance of the model is verified under various fault conditions including different fault locations and fault types. Also the suggested protection zones are illustrated in this paper. Protection of wind farm collection feeder based on directional algorithm is proposed in this paper. This algorithm for directional angle has advantages such as eliminates the need for separate relays for each wind generator, one setting file for the whole collector feeder section and so ease of engineering, monitoring the whole collector feeder section is configuration free as one relay has all the information already, discrimination between internal fault in collection feeder and external fault (grid fault) and parallel feeder, eliminate voltage signals by using voltage signal at the collection bus is reference for all feeder sections, isolate the wind WTG that connected to the cable feeder during fault at cable feeder, to completely isolate the faulted section from any electrical source. Also discrimination between cable feeder zone fault and wind turbine generator (WTG) zone fault. To verify the effectiveness of the proposed scheme it is tested in the laboratory. The experimental results indicate that it are compatible with the simulated results and the proposed technique is stable, reliable, and fast during the discrimination between internal and external faults. 


\section{REFERENCES}

[1]. Rich Hunt, Jorge Cardenas, Vijayasarathi Muthukrishnan, David McGinn, GE Digital Energy - "Multilin Wind Farm Protection Using an IEC 61850 Process Bus Architecture" Presented at the 2010 DistribuTech Conference \& Exposition, March 23, 2010

[2]. U.S. department of energy, "20\% Wind Energy by 2030 Increasing Wind Energy's Contribution to U.S. Electricity Supply", July 2008, Available electronically at http://www.osti.gov/bridge.

[3]. S. Haslam, P. Crossley and N. Jenkins, "Design and evaluation of a wind farm protection relay", Generation, Transmission and Distribution, IEE Proceedings, Volume 146, Issue 1, Jan. 1999 , pp. $37-44$.

[4]. Jones, Doug, and Kyle Bennett. "Wind farm collector protection using directional overcurrent elements." PES T\&D 2012. IEEE, 2012.

[5]. Lilik Jamilatul Awalin, Hazlie Mokhlis, Ab Halim Abu Bakar, "Recent Developments in Fault Location Methods for Distribution Networks", Przegląd Elektrotechniczny, ISSN 00332097, R. 88 NR 12a/2012.

[6]. Doug Jones, John J. Kumm, "Future Distribution Feeder Protection Using Directional Overcurrent Elements", IEEE Transactions on Industry Applications, VOL. 50, NO. 2, March/April 2014.

[7]. Lilik Jamilatul Awalin, Hazlie Mokhlis, Ab Halim Abu Bakar, "Recent Developments in Fault Location Methods for Distribution Networks", Przegląd Elektrotechniczny, ISSN 00332097, R. 88 NR 12a/2012.

[8]. Rones, V. Arthi Sahaya, and K. P. Vittal. "Adaptive protection schemes for feeders with the penetration of SEIG based wind farm." 2013 IEEE Innovative Smart Grid TechnologiesAsia (ISGT Asia). IEEE, 2013.

[9]. Jang, S. I., et al. "An adaptive relaying for the protection of a wind farm interconnected with distribution networks." 2003 IEEE PES Transmission and Distribution Conference and Exposition (IEEE Cat. No. 03CH37495). Vol. 1. IEEE, 2003.

[10]. Jorun Irene Marvikm Hans Kristian Hoidalen, Astrid PETTERTEIG, "Localization of Short-Circuits on a Medium Voltage Feeder with Distributed Generation", 20th International Conference on Electricity Distribution, Prague, 8-11 June 2009.

[11]. Connecting Wind Farms", IEEE Transactions on Energy Conversion, VOL. 22, NO. 1, March 2007.

[12]. Dr. Bhola Jha, R. B. Yadav, Dr. K. R. M Rao and H. L. Yadav, "Selection of Optimal Mother Wavelet for Fault Detection Using Discrete Wavelet Transform", International Journal of Advanced Research in Electrical, Electronics and Instrumentation Engineering Vol. 2, Issue 6, June 2013.

[13]. Nagy I. Elkalashy, Abdelsalam M. Elhaffar, Tamer A. Kawady, Naser G. Tarhuni, and Matti Lehtonen,"Bayesian Selectivity Technique for Earth Fault Protection in MediumVoltage Networks", IEEE Transactions on Power Delivery, VOL. 25, NO. 4, October 2010. 
[14]. Ahmed Hossam ELdin, Emtethal Abdallah, Nancy Mohamed,"Detection of High Impedance Faults in Medium Voltage Distribution Networks Using Discrete Wavelet Transform", 22nd International Conference on Electricity Distribution, 10-13 June 2013.

[15]. Elkalashy, Nagy I., et al. "A novel selectivity technique for high impedance arcing fault detection in compensated MV networks." European Transactions on Electrical Power 18.4 (2008): 344-363.

[16]. Mohamed F. Abdel-Fattah and Matti Lehtonen, "A Transient Fault Detection Technique with Varying Fault Detection Window of Earth Modes in Unearthed MV Systems", 978-14244-2501-3/08/2008 IEEE.

[17]. Brian McNiff, "Wind Turbine Lightning Protection Project 1999-2001" MAY 2002 National Renewable Energy Laboratory.

[18]. Abu Dhabi, "Renewable Energy Outlook: Egypt, International Renewable Energy Agency", IRENA (2018).

[19]. Ashour Abdel Salam Moussa,"NEW and Renewable Energy Authority (NREA), Cairo Egypt, Wind Energy in Egypt",DEWI Magazin Nr. 17, August 2000.

[20]. Horak, John, "Directional Overcurrent Relaying (67) Concepts", 59th Annual Conference for Protective Relay Engineers. IEEE, 2006.

[21]. R. H. Bishop" Learning with Lab-View" Book, Addison Wasley Longman, Inc. 1999. 